\title{
INTERPRETATION OF THE CO BANDS OF SUPERNOVA 1987A
}

\author{
C.M. Sharp and P. Höflich \\ Max-Planck-Institute für Physik und Astrophysik \\ Institut für Astrophsilk \\ Karl Schwarzchild Str.1, 8046 Garching, FRG
}

\begin{abstract}
Model calculations for the interpretations of the $C O$ bands in the IR are discussed. Spherical symmetry is assumed. Profiles of the physical quantities (i.e. temperature, mass, eleotron density, abundances, etc.) are given by NLTE models which allow for the representation of observed spectra of supernova 1987A in the optical and near infrared. $\mathrm{C}$ and $\mathrm{O}$ have to be chosen overabundant by a factor of 25 in the helium-rich layers, or a clumpy density distribution structure of a corresponding factor relative to the mean local value is needed. However, the comparison between the observed forbidden or line at about $6300 \mathrm{~A}$ favours the firat interpretation. A factor of about 3 has to be taken into account as uncertainty for the overabundance because of the assumptions of the becksround model. This implies evidence for atrong mixing of the helium rich region with more central layers during the stellar evolution and/or the explosion.
\end{abstract}

\section{Introduction}

Many of the properties of the supernova 1987A in the Large Magellanic Cloud are known fairly well, e.g., the progenitor star had about a 20$25 \mathrm{M}_{0}$ at the main sequence (Arnett, 1987; Hildebrandt et al., 1987; Höflich, 1987; Woosley et al., 1987).

There are, however, many questions and uncertainties related to the stellar explosion and possible mixing of different layers. Strong evidence for mixing of the $H$ and He layers and the innermost layers of the progenitor star has been found from the light ourve as well as the observed spectra (Nomoto, 1988; Woosley, 1988; Hbflich, 1988). There is also some evidence of mixing of the $C$ and $O$ region with the He shell during or after the explosion, but there are a number of difficulties in directly observing $C$ and $O$ because of the lack of umblended atomic lines and strong NLTE effects. However, the molecule 00 is measured in the infrared (IAU Circulars 4457, 4468, 4484, 4600; Danziger et al., 1987 ) which allows good determinations of the enrichment of carbon and oxygen. 
In order to investigate a possible mixing of the $\mathrm{C}$ - and O-rich layers, we have calculated the $\infty$ emigsion features. In the first section, the background model is described, followed by a discussion on how the $\infty$ opecities are computed; then its influence on the models for different abundances are considered and comparisons are made with the observed spectra.

\section{The model construction}

NLTE models of scattering-dominated photospheres of type II supernovae are used as the background models and have been applied to SN 1987A. They can be characterised by the assumption of radiative equilibrium, spherical geometry and homologeous expansion of an initial density and chemical distribution. These models allow for the representation of the observed spectra including the time dependence in the optical and nearer IR-wavelength range (see Höflich 1987, 1988 for more details of the models). The electron temperature, the particle density and chenical profile, the electron density and especially the NLTE ionization stages of CI and OI have been used in order to calculate the $\infty$ abundances and cross sections (see below) for a given model. We calculated the formation of $\infty$ by the assumption of LTE relative to CI and OI. This is a reasonable assumption because the particle densities in the C-forming regions are quite high ( $\approx 10^{11} \mathrm{~cm}^{-9}$; see Höflich, 1988), and consequently the $\infty$ formation is very fast, In the optically thin regions, it is assumed that once $\infty$ is formed, it will not be destroyed because of its high dissociation energy of $11 \mathrm{eV}$. This is a reasonable assumption because of the decrease of the collisional rates due to the decreasing density, and because of the gecmetrical dilution of the radiation field. The retroaction of the $\infty$ opacities on the NLTE model has been neglected, because most of the $C$ and $O(\leq 90 \%)$ is in CI or OI in the relevant regions of the envelope. However, a possible cooling effect of $\infty$ on the local electron temperature should be noted.

\section{Models for the $\infty$ molecule}

As $\infty$ is the most tightly bound diatomic molecule and carbon and oxygen are relatively abundant, $\infty$ is usually the second most abundant molecule in stellar atmospheres and the interstellar medium after $\mathrm{H}_{2}$. The IR vibration-rotation spectrum of $\infty$ is one of the simplest molecular spectra, so the monochromatic absorption coeffioient can be rapidly computed using approximate techniques that are sufficiently accurate for our purposes here.

Due to its high dissociation energy, the Morse approxtmation is good for many of the vibrational levels. With this method the first order anharmonic correction to the harmonic oscillator is allowed for, with higher order corrections being neglected, and an efficient analytic approximation is used to calculate the vibrational band strengths (Sharp, 1988). However, higher onder anharmonicity is allowed for in computing the band frequencies. Fortunately, as the ground electronic state of $C O$ is ${ }^{1 \Sigma} \Sigma$, the rotational fine structure of each band consists of only a single P-branch $(\Delta J=-1)$ and $R$ branch $(\Delta J=+1)$. The total line strength is obtained from the Boltzmann factor for the temperature cor- 
responding to the colligional temperature, partition function, vibrational band strength and the rotational Hönl-London factor. Non-rigidity and the coupling of vibration and rotation is allowed for in calculating the rotational levels and line frequencies, but is neglected in determining the line strengths. Each rotational line is broadened with a Gaussian profile dependent on the temperature and some assumed turbulent velocity.

The region of the spectrum that is of interest is divided up into several thousand equally apaced intervals of widths approximately conparable to the line widths. Each vibration-rotation band is computed in a progression starting with the fundanental and its "hot" bands, i.e. $\Delta v=1$, then the first overtone and its "hot" bands, i.e. $\Delta v=2$ etc. In general each profile will cover several intervals, and using the error function, the mean absorption in each interval is obtained.

\section{Discusgion of the synthetic spectra and applications to the SN}

An atmospheric model is used as the backsround which allows for the representation of the optical and near-infrared spectra observed on 2 October 1987 ( $\left.T_{* f}\right)=4900 \mathrm{~K} ; R_{\text {flownlu }}=7.810^{14} \mathrm{~cm}$; particle density

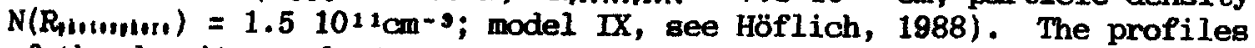
of the density, eleotron temperature and ionization and of the excitation stages of all ions are assumed to be given by this NLTE model. The typical tempweratures in the $\infty$-forming region are in the order of 4000 to $1500 \mathrm{~K}$.

The influence of the carbon and oxgren abundances on the emitted IR spectra are investigated (Figure 1). The sensitivity of the band strength on the abundances should be noted. Below abundance of about 10 times solar, the lst overtone system of $\infty$ can be assumed to be optically thin. However, note the finite optical thickness for higher

Figure 1: The synthetic IR spectra labelled by the assumed over-abundance of $\mathrm{C}$ and $\mathrm{O}$ relative to the solar photosphere are given as calculated for the background model (see text).

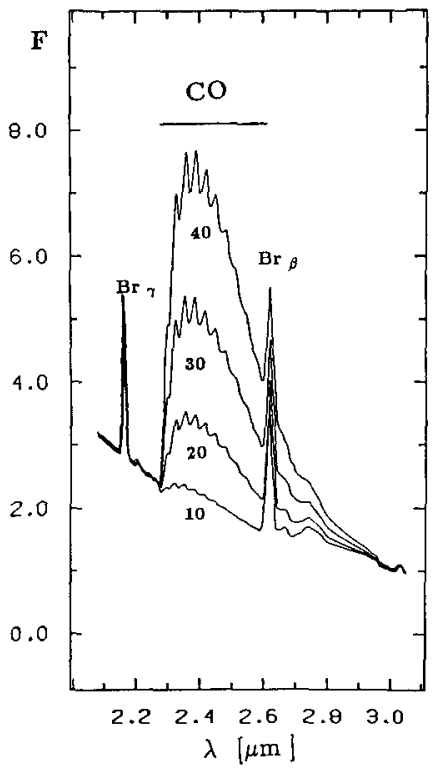


Figure 2: The optical depths of the first overtone system of $\infty$ at the Thompson optical depth of 1 (curve 1) and the maximum ratio of $\infty /(C+\infty)$ (curve 2) are given as a function of the abundance relative to hydrogen in the solar photosphere as calculated for the background model (see text).

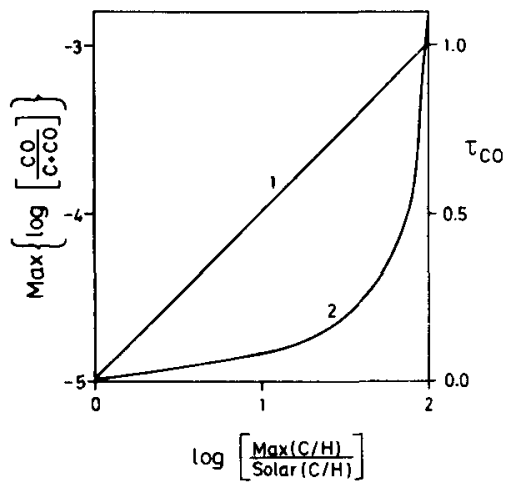

abundances. The first overtone bands are formed near the stellar photosphere in the parameter range we are interested in. Therefore, the temperatures in these layers are not strongly affected by the cooling due to this molecule.

However, the fundamental system is formed at much larger distances at which the temperature is influenced by molecular cooling. Therefore, we restrict our discussion here to the overtone bands. Note, in addition, that most of the carbon and oxycten are in the atomic states (curve 2, Figure 2). Therefore, the $\infty$-particle density depends on the particle density of $\mathrm{C}$ and $\mathrm{O}$ only. The given abundances may also be interpreted as a locally higher density, e.f., the overabundance of 25 of $C O$ can also be produced by local density fluctuations of the corresponding order. This would have only mall influence on the calculated spectra in the optical wavelength range.

A comparison of the observed and calculated spectra (Fioure 3) show good agreement for an overabundance in the order of 25 times solar in the layers just above the photosphere. This overabundance may either be interpreted as mixing of these layers with the carbon- and oxygenrich layers that were originally located somewhat deeper in the star, or this may be due to density fluctuations. However, the observed forbidden oI line in the optical wavelength range favours the first explanation as the main effect (Höflich, 1988).

Figure 3: IR spectrum as observed by ESO between 1 and 6 oct. 1987 (upper curve shifted by two units) in comparison with the reddened, synthetic spectrum (lower curve, $25 *$ solar $C$ and $\left.O, E_{-v}=0.15\right)$. In addition, some line identifications are given.

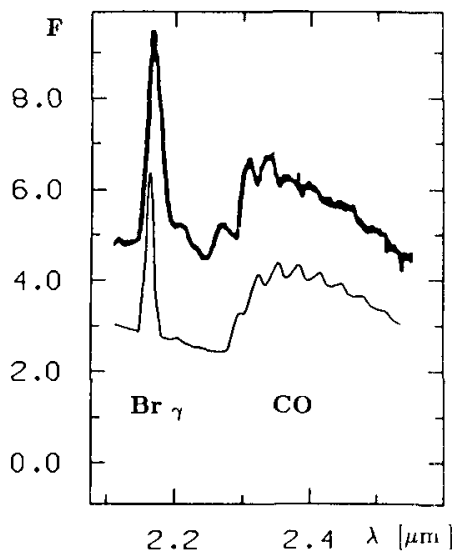




\section{Sumary and conclugions}

We have demonstrated that the $C O$ emission of SN1987A as observed after about 7 months is well explained by a model which allows for the representation of the optical spectra if a higher particle density of carbon and oxygen by a factor of about 25 is assumed. This may be either due to an overabundance of $\mathrm{C}$ and $\mathrm{O}$ in the helium rich layers or due to local temporary density fluctuations. However, the optical spectrum of the forbidden oI feature favours the first interpretation, and therefore indicates some mixing of the helium rich stellar layers with those below. Additionally, we already know that the $\mathrm{H}$ and He layers as well as the inner layers are mixed (Nomoto, 1988; Woosley, 1988; Höflich, 1988a). We can conclude that all layers have to undergo some mixing and a detailed determination of the chemical profiles by the observed spectra may give important information concerning the typical mixing scales. However, the observed OI line profiles (Dopita, personal communication, 1988), the FeII and CoII lines indicate some inhomogeneities of the density structure (Ericson, personal communication; Hilldebrandt et al., 1988). Therefore, some contribution caused by the clumpiness of the matter of our $C O$ determination has to be expected.

We have also to stress the limitations of our anaylses. The assumption of the negligible retraaction of the cooling of the molecules for the relevant regions certainly breaks down if the corresponding features are formed well above the photosphere. This is true in these conditions for the fundamental bands. Therefore, a discussion of the line ratios of the different systems needs consistent models (including the molecular cooling). Such models will enable us to determine the chemical profiles of $\mathrm{C}$ and $\mathrm{O}$ more accurately. This will be discussed in a forthcoming paper.

\section{References}

Arnett, W.D. 1987, Astrophys. J., 319,136.

Danziger, I.J., Bouchet, P., Fosbury, R.A.E., Gouiffes, C. ; Lucy, L.B. , Moorwood, A.F.M., Oliva, E., and Rufener, F. 1987. George Mason Conference, October 1987.

Hilldebrandt, W., Höflich, P., Truran, J.W., and Weiss, A. 1987, Nature, $327,597$.

Hilldebrandt, W., Höflich, P., Janka, H.- T., and Monchmeyer, R. 1988.

To appear in Proceedings of the ESO-CERN Workshop, Jume 1988.

Höflich, P. 1987, in "ESO-workshop on SN1987A", ed. I.J.Danziger, Garching, p. 447 .

Höflich, P. 1988, in Proceedings of the Astronomical Society of Australia, Canberra, June 1988, in press.

Nomoto, K. 1988, preprint.

Sharp, C.M. 1988, Astron. Astrophys. Suppl. Ser, 72, 355.

Woosley, S.E. 1988, preprint.

Woosley, S.E., Pinto, P.A., and Ensman,L. 1987, Astrophys.J., 324, 466. 